\title{
Análise de conceito do resultado de enfermagem Mobilidade em pacientes com acidente vascular cerebral
}

\author{
Concept analysis of the nursing outcome Mobility in patients with stroke \\ Conceptual análisis del resultado de enfermería Movilidad en pacientes con accidente cerebrovascular
}

\section{Rafaella Pessoa Moreira', Thelma Leite de Araujo", Tahissa Frota Cavalcante', Nirla Gomes Guedes", Marcos Venicios de Oliveira Lopes", Emília Soares Chaves'}

' Universidade da Integração Internacional da Lusofonia Afro-Brasileira, Curso de Enfermagem. Fortaleza-CE, Brasil.

"Universidade Federal do Ceará, Departamento de Enfermagem. Fortaleza-CE, Brasil.

\author{
Submissão: 27-09-2012 Aprovação: 18-02-2014
}

\section{RESUMO}

O objetivo foi realizar análise do conceito do resultado de enfermagem Mobilidade em paciente com AVC. Realizou-se revisão integrativa da literatura, por meio de acesso on-line às bases: Scopus, Pubmed, Cinahl, Cochrane e Lilacs, usando-se os descritores: mobility, stroke, nursing e sinonímias nas línguas portuguesa e espanhola. Identificaram-se 1.521 artigos de que, após seleção criteriosa, resultaram 49. Destacam-se artigos publicados no Canadá (26,7\%), a partir de 2001 (95,9\%), por fisioterapeutas (34,6\%), em unidades de reabilitação (61,5\%). Atributos identificados para Mobilidade: andar, ficar em pé, sentar, colocar a perna de um lado para outro, virar-se, iniciar e parar a locomoção, subir escadas, função motora, transferência e habilidade motora. Também foi construído caso modelo, caso contrário e identificados antecedentes: controle postural e equilíbrio; e consequentes: realiza tarefas no interior e exterior da casa e deambula sem dificuldade. Os conceitos do resultado Mobilidade encontrados nesta pesquisa precisam ser validados com especialistas na área e na prática clínica.

Descritores: Enfermagem; Acidente Cerebral Vascular; Avaliação de Resultados.

\begin{abstract}
This study aimed a concept analysis of the nursing outcome Mobility in patients with stroke. A literature review was conducted, through the online access to databases: Scopus, Pubmed, CINAHL, Cochrane, and Lilacs, using the descriptors: mobility, stroke, nursing and their synonyms in Portuguese and Spanish. 1.521 articles were identified, resulting in 49, after careful selection. Noteworthy are the articles published in Canada (26.7\%), on 2001 (95.9\%), by physiotherapists (34.6\%), and in rehabilitation units (61.5\%). The attributes identified for Mobility were: walking, standing, sitting, put the leg side to side, turn around, start and stop walking, stair climbing, motor function, and motor skill transfer. A model case and a contrary case were built, and identified, as antecedents: postural control and balance; and, as consequents: performs tasks inside and outside the house and wanders without difficulty. It was concluded that the concepts of Mobility found in this study need to be validated with experts in the field and in clinical practice.
\end{abstract}

Key words: Nursing; Stroke; Outcome Assessment.

\section{RESUMEN}

Objetivó-se realizar un análisis conceptual del resultado de enfermería Movilidad en pacientes con accidente cerebrovascular. Se realizó una revisión de la literatura, con el acceso a bases de dados: Scopus, PubMed, CINAHL, Cochrane, Lilacs, usandose los descriptores: movilidad, accidente cerebrovascular y enfermería, y sus sinónimos en portugués y español. Fueron identificados 1.521 artículos, y seleccionados 49. Los artículos fueron publicados en Canadá (26,7\%), en 2001 (95,9\%), por fisioterapeutas $(34,6 \%)$, y en unidades de rehabilitación $(61,5 \%)$. Los atributos identificados fueran: caminar, pararse, sentarse, poner el lado de la pierna a otro, dar la vuelta, empezar y dejar de caminar, subir escaleras, la función motora y la transferencia de habilidades motoras. Se construyó un caso modelo y un caso contrario. Antecedentes identificados: el control postural y el equilibrio. Consecuentes: realiza tareas dentro y fuera de la casa y vagar sin dificultad. Los conceptos del resultado de enfermería Movilidad de ingresos identificados en este estudio necesitan ser validados con expertos en el campo y en la práctica clínica.

Palabras clave: Enfermería; Accidente Cerebrovascular; Evaluácion de Resultado. 


\section{INTRODUÇÃO}

Neste estudo, teve-se por objeto a análise do conceito do resultado de enfermagem Mobilidade em pacientes que sobreviveram ao acidente vascular cerebral (AVC). A delimitação do estudo em pessoas com esta doença encontra respaldo na grande incidência e consequências do AVC, já que é a primeira causa de incapacidade e a segunda causa de mortalidade no mundo ${ }^{(1)}$, fator de preocupação para o cuidado de enfermagem. O mencionado resultado foi estabelecido com base na Classificação dos Resultados de Enfermagem - NOC (2).

O aprimoramento do resultado de enfermagem é importante para a prática clínica, tendo em vista que o enfermeiro ao utilizar o processo de enfermagem, durante o cuidado a um paciente, deve, após o levantamento de dados, por meio de entrevista e exame físico, identificar os diagnósticos de enfermagem e planejar quais resultados desejam ser alcançados para traçar intervenções eficazes.

Para a concretização deste estudo foi realizada a análise de um conceito. Destaca-se que o conceito é uma ideia ou construção mental elaborada acerca de um fenômeno, sendo essencial no desenvolvimento de pesquisas, contribuindo para prática baseada em evidência. Os conceitos compreendem atributos abstratos da realidade.

Para estudar um conceito é necessária a utilização de um Modelo de Análise de Conceito. Conforme recomenda Hoskins $^{(3)}$, a análise de conceito deve ser efetivada para identificar seus atributos particulares e característicos e indica, para tanto, os procedimentos descritos por Walker e Avant ${ }^{(4)}$. Estas autoras propõem uma análise modificada e simplificada com base em um modelo clássico de análise de conceito anteriormente sugerido por Wilson ${ }^{(5)}$. Para a realização do referido estudo, adotou-se o referencial de Walker e Avant ${ }^{(4)}$, porquanto, segundo as autoras, o seu modelo tem referência direta com a enfermagem e mais especificamente com os sistemas de classificação da linguagem da enfermagem.

Para desenvolver as etapas da análise de conceito propostas por Walker e Avant( ${ }^{(4)}$ é indispensável ampliar a busca na literatura. Estudos afirmam a necessidade de uma revisão de literatura sólida, específica, com vistas a identificar as teorias que subjazem ao construto e embasar a formulação de cada item da ferramenta em construção ${ }^{(6)}$.

Como método para a revisão de literatura do presente estudo, seguiram-se os passos da revisão integrativa proposta por Whittemore e Knafl(7), a qual sintetiza resultados de pesquisas relevantes e reconhecidos mundialmente. Desse modo, facilita a incorporação de evidências, ou seja, agilizando a transferência de conhecimento novo para a prática.

A relevância de pesquisas como esta, alicerça-se na escassez de estudos de análise de conceito e na compreensão da necessidade de refinamento do resultado de enfermagem em populações específicas, aqui exemplificadas pelos indivíduos com acidente vascular cerebral.

A realização deste estudo partiu do seguinte questionamento: será que a definição do resultado de enfermagem Mobilidade proposta pela NOC $^{(2)}$ é adequada para pacientes com acidente vascular cerebral?
Diante disso, o objetivo deste estudo foi realizar análise do conceito do resultado de enfermagem Mobilidade em pacientes que sobreviveram ao acidente vascular cerebral.

\section{MATERIAIS E MÉTODOS}

No intuito de auxiliar a execução desta etapa foram adotados o Modelo de Análise de Conceito proposto por Walker e Avant ${ }^{(4)}$ e a Revisão Integrativa da Literatura de Whittemore e Knafl(7) ${ }^{(7)}$ fonforme proposto por Hoskins ${ }^{(3)}$. Apesar de terem itens semelhantes, eles estão descritos separados para maior clareza.

\section{Revisão Integrativa da Literatura}

Com vistas a guiar a primeira etapa da revisão integrativa foram formuladas estas questões: qual a definição de Mobilidade? Quais os antecedentes, atributos e consequentes de Mobilidade?

Como referido, os artigos identificados foram buscados em uma população específica de pacientes com acidente vascular cerebral. Para a seleção das produções adotou-se o acesso on-line a cinco bases de dados: Scopus, National Library of Medicine and National Institutes of Health (Pubmed), Cumulative Index to Nursing and Allied Health Literature (Cinahl), Cochrane e Literatura Latino-Americana em Ciências de Saúde (Lilacs). Mediante essas diversas bases de dados tentou-se ampliar o âmbito da pesquisa e dessa forma minimizar possíveis vieses. A etapa de busca na base de dados ocorreu nos meses de maio e junho de 2010.

Na busca às bases de dados Pubmed e Cinahl, usou-se a terminologia preconizada, conforme o vocabulário Medical Subject Headings of U.S (MeSH). National Library of Medicine em língua inglesa. Os descritores controlados utilizados foram: Mobility; Stroke; Nursing. Para as demais bases de dados, adotou-se o vocabulário estruturado DeCS - Descritores em Ciências da Saúde. Contudo na base de dados Lilacs, o termo utilizado para a busca dos estudos é o próprio descritor e nas outras bases de dados (Scopus e Cochrane) os descritores são conhecidos como palavras-chave ou termos. Assim, os descritores empregados para a busca nas bases de dados Lilacs, Scopus e Cochrane foram estes: na língua portuguesa (Mobilidade; Acidente Cerebral Vascular; enfermagem), na inglesa (Mobility; Stroke; nursing); e na espanhola (Movilidad; Accidente Cerebrovascular; Enfermería). Ressalta-se que, na base de dados Scopus, foram utilizados descritores não controlados.

Quanto aos critérios de inclusão, foram: ser artigos; estar disponíveis eletronicamente nos idiomas português, inglês ou espanhol; abordar a temática Mobilidade em pacientes com acidente vascular cerebral; ser realizado com pessoas com idade mínima de 18 anos; responder à questão norteadora deste estudo. Excluíram-se, porém, os editoriais, cartas ao editor, estudos reflexivos, relatos de experiência, projetos e publicações duplicadas.

Ressalta-se que a busca nas bases de dados aconteceu ao mesmo tempo, mas de forma individual, pela pesquisadora e por uma bolsista de apoio técnico, treinada para esta 
atividade. Esta estratégia foi seguida com vistas a garantir que os artigos selecionados atendessem aos critérios de inclusão. Ademais, o acesso em cada base de dados foi efetuado em um único dia. Após identificação dos artigos encontrados, foram salvos os títulos e os resumos em documento do Word para evitar que, ao acessar em outro dia, ocorresse mudança na quantidade de artigos. Em seguida, foram lidos todos os títulos e resumos para saber quais artigos atendiam aos critérios de inclusão. Dessa forma, fez-se comparação entre os resultados da pesquisadora e da bolsista. Em caso de diferenças, tentava-se definir se o artigo seria incluído ou não, mediante uma análise mais profunda do material. Em seguida, apenas a pesquisadora procedeu à busca na íntegra dos artigos, excluindo aqueles que não estavam disponíveis eletronicamente.

Foram identificados 1.521 artigos em todas as bases de dados pesquisadas e, após a seleção por meio de título e resumo, restaram 388. Estes foram impressos para uma primeira leitura. A partir de então, excluíram-se aqueles que não abordavam a temática de modo a alcançar o objetivo do estudo, os quais não tinham sido excluídos apenas pela leitura do título e do resumo.

Assim, 103 artigos foram analisados mais profundamente na segunda leitura. Dessa vez, excluíram-se os que não trouxeram conceitos importantes para construção do trabalho. Procedeu-se, a seguir, à terceira leitura detalhada de 61 artigos. Desses, doze foram excluídos por não trazerem conceitos relevantes. Ao final, totalizaram 49 artigos de todas as bases de dados, sendo 14 (Scopus), 15 (Pubmed), 15 (Cinahl), 4 (Cochrane) e 1 (Lilacs).

Para a avaliação dos estudos selecionados adotou-se a proposta de Melnykee, Fineout-Overholt ${ }^{(8)}$, os quais classificam os estudos segundo as forças de evidências.

As informações extraídas dos artigos foram identificadas e documentadas, sendo a avaliação e análise dos dados realizada no período de julho de 2010 a janeiro de 2011 .

\section{Modelo de Análise do Conceito de Walker e Avant}

As etapas do Modelo de Análise do Conceito ${ }^{(4)}$ são: seleção do conceito; determinação dos objetivos e propostas para análise conceitual; identificação dos possíveis usos do conceito; determinação dos atributos definidores; identificação de um caso modelo; identificação de casos borderline, contrários, inventados e ilegítimos; identificação dos antecedentes e consequentes do conceito; definição de referentes empíricos. Ressalta-se que algumas etapas do Modelo de Análise do Conceito ${ }^{(4)}$ são equivalentes e ocorrem de forma simultânea às etapas da Revisão Integrativa da Literatura ${ }^{(7)}$, tais como: seleção do conceito; determinação dos objetivos da análise conceitual; e identificação dos possíveis usos do conceito.

Após a leitura dos 49 artigos, identificaram-se os atributos críticos do conceito de Mobilidade, assim como os seus antecedentes e consequentes. Também foram criados casos-modelos e casos-contrários ${ }^{(4)}$.

Inicialmente será detalhada a caracterização dos artigos e em seguida, os resultados serão apresentados, conforme os passos da análise de conceito empreendida, exceto a descrição das referências empíricas por não ser necessária para contemplar o objetivo proposto.

A pesquisa não foi submetida ao Comitê de Ética em Pesquisa pelo fato de tratar de uma análise de conceito com busca apenas na literatura.

\section{RESULTADOS}

Entre os quinze diferentes países identificados nos artigos analisados, destacam-se o Canadá e os Estados Unidos da América com $26,7 \%$ e $16,4 \%$ das produções, respectivamente. No tocante ao ano de publicação, com exceção de dois artigos datados de 1999, os demais foram publicados a partir do ano de 2001. Em 2009, obteve-se o maior número $(16,4 \%)$.

Os profissionais responsáveis pelos artigos eram, na sua maioria, fisioterapeutas $(34,6 \%)$, seguidos por médicos $(32,6 \%)$. Também houve um considerável número de publicações de equipes multiprofissionais (22,5\%). Apenas dois artigos eram de autoria exclusiva de enfermeiros.

$\mathrm{Na}$ maior parte dos artigos os dados foram coletados em unidades de reabilitação $(61,5 \%)$. O domicílio e os hospitais também foram cenários de coleta de dados referidos, respectivamente, em $12,0 \%$ dos artigos analisados. Em $14,5 \%$ não se definiu o cenário do estudo por se tratar de pesquisas de revisão, incluindo estudos desenvolvidos em diferentes locais.

Prevaleceu nos artigos a temática relacionada a programas de exercícios para melhorar a mobilidade $(40,8 \%)$, seguida pelas consequências decorrentes do AVC (28,6\%), tais como: problemas urinários, úlceras por pressão, dor, problemas ósseos, alteração de equilíbrio e queda da própria mobilidade em pacientes com AVC.

Entre os estudos avaliados, destacaram-se as pesquisas descritivas ou qualitativas $(69,5 \%)$ relativas ao nível $\mathrm{VI}$ de evidência. Também estiveram presentes os ensaios clínicos randomizados bem delineados, nível de evidência II (10,2\%), assim como os estudos de coorte ou caso-controle bem delineados, com nível de evidência IV (10,2\%). E, apesar de em menor quantidade, é relevante o fato de o presente estudo incluir artigos de revisão sistemática/metanálise $(6,1 \%)$. No entanto, não foi identificado nenhum estudo de ensaio clínico bem delineado sem randomização, correspondente ao nível de evidência III.

- Seleção do conceito, determinação dos objetivos ou fins da análise do conceito, e identificação de usos dos conceitos de Mobilidade

Vale destacar que inicialmente para realização da análise do conceito, selecionou-se o conceito Mobilidade. Nesta etapa, os objetivos foram: realizar a análise de conceito do termo Mobilidade para identificar os usos (definições), os atributos críticos, os possíveis antecedentes e os consequentes de Mobilidade. No Quadro 1 expõem-se as definições identificadas na literatura para o uso do termo Mobilidade. 
Quadro 1 - Definições para o termo Mobilidade identificadas na literatura. Fortaleza-CE, 2011

\begin{tabular}{|c|}
\hline DEFINIÇÕES \\
\hline Um meio viável de locomoção ${ }^{(9)}$. \\
\hline $\begin{array}{l}\text { Capacidade do indivíduo de se mover de forma eficaz no seu am- } \\
\text { biente }^{(10)} \text {. }\end{array}$ \\
\hline $\begin{array}{l}\text { Capacidade de se movimentar de um local para outro e exige tanto } \\
\text { a função motora como a capacidade de passar de uma posição para } \\
\text { outra ou de um local para outro }{ }^{(11)} \text {. }\end{array}$ \\
\hline $\begin{array}{l}\text { Movimento da pessoa de uma posição postural para outra ou de um } \\
\text { local para outro }{ }^{(12)} \text {. }\end{array}$ \\
\hline Movimento físico independente dentro do ambiente ${ }^{(13)}$. \\
\hline
\end{tabular}

\section{- Determinação dos atributos definidores de Mobilidade}

O atributo é o que mais está associado ao conceito. São as palavras ou expressões utilizadas pelos autores para descrever as características do conceito. Permite ao analista ampla visão sobre o conceito. Os atributos podem modificar a compreensão do conceito ou melhorá-la. Este pode mudar ao longo do tempo ou quando usado em um contexto diferente do pesquisado ${ }^{(4)}$.

Para a identificação dos atributos críticos concernentes à Mobilidade em pacientes com acidente vascular cerebral, utilizaram-se as seguintes questões: Como os autores definem o conceito? Quais as características ou atributos apontados? Que ideias os autores discutem quanto à Mobilidade em pacientes com AVC?

Quadro 2 - Atributos de Mobilidade identificados na literatura. Fortaleza-CE, 2011

\begin{tabular}{|l|}
\hline \multicolumn{1}{|c|}{ ATRIBUTOS } \\
\hline $\begin{array}{l}\text { Andar, ficar em pé, sentar, colocar a perna de um lado para outro, } \\
\text { virar-se, iniciar e parar a locomoção, subir escadas }\end{array}$ \\
\hline Função motora $^{(13)}$. \\
\hline Transferência e habilidade motora $^{(15)}$. \\
\hline
\end{tabular}

- Identificação de um caso modelo e de um caso contrário para Mobilidade

\section{Caso modelo Mobilidade}

Sra. Lúcia, 60 anos, aposentada. Todos os dias ao acordar, espreguiça-se, mexe as pernas de um lado para outro, em alongamentos. Em seguida, levanta-se da cama e vai até o banheiro para escovar os dentes. Depois, inicia as tarefas domésticas: varre a casa, passa o pano, cozinha e lava as louças. Ao final do dia sai de casa sozinha, sobe as escadas da igreja, senta nos bancos em frente ao altar e passa vinte minutos em orações.
Depois, desce as escadas e vai para a praça onde caminha durante trinta minutos. A Sra. Lúcia está com a saúde muito boa.

Ressalta-se que este caso modelo é fictício e contempla os atributos identificados na literatura para o termo Mobilidade, tais como: andar, ficar em pé, sentar, colocar a perna de um lado para outro, virar-se, iniciar e parar a locomoção, subir escadas, transferir e habilidade motora.

\section{Caso-contrário de Mobilidade}

Sr. Carlos, 28 anos, era uma pessoa muito ativa. Praticava esportes radicais, até sofrer um acidente vascular cerebral quando é levado inconsciente para o hospital. Após submeter-se a vários exames, os médicos referem que ele perdeu os movimentos do lado direito do corpo. Meses depois da cirurgia, o paciente não consegue ficar em pé sozinho, caminhar e subir escadas. Torna-se, então, dependente de outras pessoas e de dispositivos auxiliares.

Este caso-contrário fictício contradiz os atributos críticos essenciais identificados para o termo Mobilidade. O paciente Carlos após um acidente vascular cerebral não mais recupera a mobilidade. Ele não consegue mais ficar em pé, caminhar e subir escadas.

Após a análise dos conceitos, identificação dos atributos críticos e construção dos casos modelo e contrário relacionados ao conceito de Mobilidade construiu-se uma definição que contempla os resultados da análise conceitual.

A definição construída para Mobilidade foi esta: capacidade da pessoa movimentar-se de uma posição postural para outra ou de um local para outro, subir e descer escadas, de forma independente, com utilização ou não de dispositivo auxiliar, como a bengala.

Enfatiza-se: esta definição difere em parte da proposta pela NOC $^{(1)}$ para o resultado Mobilidade: capacidade de movimentar-se propositadamente pelo ambiente, de forma independente, com ou sem dispositivo auxiliar.

\section{- Identificação dos antecedentes do conceito}

Os eventos antecedentes são os acontecimentos ou incidentes que devem aparecer antes da ocorrência do conceito $^{(4)}$. Estes foram identificados por meio da resposta à pergunta: que eventos, situações e/ou fenômeno contribuem para a evidência do conceito de Mobilidade em pacientes com AVC?

No Quadro 3 expõem-se o antecedente identificado.

Quadro 3 - Antecedente de Mobilidade identificado na literatura. Fortaleza-CE, 2011

\begin{tabular}{|c|}
\hline ANTECEDENTE \\
\hline Controle postural e equilíbrio ${ }^{(9,16-17)}$ \\
\hline
\end{tabular}

\section{- Identificação dos consequentes do conceito}

Os consequentes do conceito são os fatos que acontecem como resultado da ocorrência do conceito ${ }^{(4)}$. Para identificação 
dos consequentes do conceito indagou-se: quais são os eventos ou situações resultantes da Mobilidade em pacientes com AVC? No Quadro 4 estão apresentadas as respostas identificadas na literatura para os consequentes da mobilidade.

Quadro 4 - Consequentes de Mobilidade identificados na literatura. Fortaleza-CE, 2011

\begin{tabular}{|l|}
\hline \multicolumn{1}{|c|}{ CONSEQUENTES } \\
\hline Realiza tarefas no interior e exterior da casa $^{(10)}$. \\
\hline Deambula sem dificuldade $^{(10)}$. \\
\hline
\end{tabular}

\section{DISCUSSÃO}

Ao examinar os artigos selecionados utilizados como base para a análise do conceito de pacientes com acidente vascular cerebral, observou-se a diversidade de locais das publicações com a temática do estudo, destacando-se os países da América do Norte. No tocante ao ano de publicação, quase todos os artigos são da década de 2000, com crescente aumento após o ano 2001. O ensino de pós-graduação foi um dos fatores determinantes do desenvolvimento da área da saúde, contribuindo para a construção do conhecimento ${ }^{(18)}$.

Consoante evidenciado, os autores eram na sua maioria fisioterapeutas e médicos. Em alguns artigos estes eram de diversas áreas da saúde. Apenas dois foram publicados exclusivamente por enfermeiros. Muitos dos resultados sensíveis à enfermagem não são específicos apenas para intervenções de enfermagem e, portanto, podem ser usados para avaliar o cuidado oferecido por outras disciplinas na área da saúde. Por exemplo, os fisioterapeutas podem exercer profunda influência sobre o nível de Mobilidade de determinados pacientes. Nesse caso, esse resultado mede os resultados colaborativos do cuidado de enfermagem e da fisioterapia. Embora os resultados possam ser empregados por outras disciplinas, os indicadores listados para avaliar a condição do paciente em relação ao resultado podem variar de disciplina para disciplina. Assim, os fisioterapeutas podem usar indicadores que mensurem o progresso com o uso de equipamentos não adotados rotineiramente por enfermeiros ${ }^{(2)}$.

Dessa forma, a parceria entre o enfermeiro e outros profissionais é válida, pois este executa seu trabalho em equipe, e a troca de experiência na área da saúde é marcante. Neste âmbito, cada um pode intervir no que compete à sua área e o resultado do conjunto de atividades desenvolvidas por todos os profissionais pode contribuir para a melhoria da qualidade da assistência. Assim, os pacientes com AVC que têm dificuldade de mobilidade poderão ser beneficiados se houver uma equipe multiprofissional cuidando da sua reabilitação.

$\mathrm{Na}$ maior parte dos artigos os dados foram coletados em unidades de reabilitação, com temática prevalente acerca dos programas de exercícios para melhorar a mobilidade, os quais normalmente são desenvolvidos nessas unidades. Nas últimas décadas houve um declínio dos registros do risco de óbito por
AVC, o qual se dá de forma diferenciada entre as populações. Isto, porém, pode colaborar para o aumento da sua prevalência, porquanto não houve declínio de mesma proporção em sua incidência em todo o mundo ${ }^{(19)}$. Diante disso, os pacientes sobreviventes ao AVC precisam participar da reabilitação e iniciar programas de exercícios para melhorar a mobilidade logo após a doença, sobretudo porque a maior parte exibe deficiências neurológicas e incapacidades residuais significativas.

Entre os estudos avaliados, destacaram-se as pesquisas descritivas ou qualitativas inerentes ao nível VI de evidência. Estas pesquisas não representam evidências clínicas fortes, no entanto, são importantes por fornecerem elementos conceituais para a análise da Mobilidade em pacientes com acidente vascular cerebral e, por tal motivo, foram adotadas.

Foram identificadas definições de Mobilidade. A mobilidade é um conceito complexo que consiste de atributos que nem sempre são diretamente evidentes ${ }^{(10)}$. Recuperar a mobilidade é talvez o principal objetivo do paciente após o AVC. Seu grau de mobilidade depende de quais movimentos são possíveis após uma sequência de acidentes vasculares cerebrais e das barreiras ambientais ${ }^{(13)}$.

De acordo com alguns autores, a mobilidade é a capacidade do indivíduo de se mover no seu ambiente ${ }^{(10)}$. O paciente se movimenta ao andar, levantar, sentar, transferir-se ${ }^{(14)}$. Estes foram alguns dos atributos identificados para mobilidade no presente estudo.

Além destes, destaca-se a função motora considerada um determinante da mobilidade em pacientes com AVC. Muitas vezes, a função motora é descrita em termos de movimento sinérgico. No entanto, em situações da vida real, o sujeito precisa de uma função motora complexa, com vistas a executar uma atividade de mobilidade ${ }^{(13)}$.

Ao analisar os antecedentes para Mobilidade, identificaram-se o controle postural e o equilíbrio. Para alguns autores, os termos "equilíbrio", "reações de equilíbrio", "reações posturais", "controle postural" e "postura" são usados indistintamente; não há definições comumente aceitas para estes termos nem coerência na forma com que eles são usados ${ }^{(20)}$.

De acordo, porém, com outros autores, estratégias motoras posturais são as organizações de movimentos adequados para controlar a posição do corpo no espaço, levando o indivíduo a desenvolver estratégias de equilíbrio antes mesmo da ocorrência de um evento capaz de perturbá-lo ${ }^{(16)}$.

Controle postural também tem sido definido como o ato de realizar, manter ou restaurar um estado de equilíbrio durante uma postura ou atividade. O controle postural é uma interação entre o indivíduo, a atividade e o ambiente. Deficiências em decorrência do AVC, tais como fraqueza muscular, tônus muscular anormal, propriocepção distorcida e comprometimento do mecanismo vestibular, podem afetar o equilíbrio ${ }^{(17)}$.

O controle postural envolve o controle da posição do corpo no espaço para estabilidade e orientação. Define-se orientação postural como a capacidade de manter uma relação adequada entre os segmentos do corpo e entre o corpo e o ambiente para determinada tarefa. Inclui também a estabilidade postural ou equilíbrio. Além disso, o equilíbrio é um pré-requisito para todas as atividades funcionais: sentar-se, ficar em pé e andar ${ }^{(16)}$. 
Consequente da Mobilidade, a deambulação tem sido amplamente definida como locomoção em diversos ambientes. Pessoas com mobilidade podem desempenhar atividades no interior da sua casa, ir a supermercado, shopping center, bancos, enfim, fazer passeio social com mais facilidade do que aqueles que têm dificuldade na locomoção(10).

Conforme recomendado, pacientes com AVC devem iniciar a mobilidade o mais precocemente possível (sentar-se, andar, transferir-se) no intuito de evitar ou diminuir a limitação da mobilidade muitas vezes imposta pela doença ${ }^{(21)}$.

\section{CONCLUSÃO}

Durante a realização da análise de conceito do resultado Mobilidade identificou-se como atributos para Mobilidade: andar, ficar em pé, sentar, colocar a perna de um lado para outro, virar-se, iniciar e parar a locomoção, subir escadas, função motora, transferência e habilidade motora. Também se construíram um caso-modelo e um caso-contrário para Mobilidade. Os antecedentes identificados para Mobilidade foram: controle postural e equilíbrio e os consequentes foram: realiza tarefas no interior e exterior da casa e deambula sem dificuldade.
A partir disso, propõe-se a modificação da definição do resultado Mobilidade proposta pela NOC em 2010 para a seguinte: capacidade da pessoa de movimentar-se de uma posição postural para outra ou de um local para outro, subir e descer escadas, de forma independente, com utilização ou não de dispositivo auxiliar, como a bengala.

A realização deste estudo pode contribuir para o refinamento e o aprimoramento do resultado de enfermagem Mobilidade presente na NOC de 2010. No entanto, recomenda-se a realização de novos estudos com essa temática, com o escopo de aprofundar e difundir o conhecimento sobre esse resultado. Fundamental é, portanto, a realização da validação por especialistas e da validação clínica para a confirmação dos resultados deste estudo.

Também é necessária a realização de outras pesquisas com o intuito de criar definições constitutivas e operacionais para os indicadores do resultado de enfermagem Mobilidade, tendo em vista a seguinte hipótese: a falta de definições constitutivas e operacionais para cada resultado e para seus indicadores interfere na uniformidade da avaliação do enfermeiro que atua na reabilitação do paciente com dificuldade na mobilidade, como é o caso dos pacientes com acidente vascular cerebral.

\section{REFERÊNCIAS}

1. Lozano R, Naghavi M, Foreman K, Lim S, Shibuya K, Aboyans $\mathrm{V}$, et al. Global and regional mortality from 235 causes of death for 20 age groups in 1990 and 2010: a systematic analysis for the Global Burden of Disease Study 2010. Lancet. 2012 Dec 15;380(9859):2095-128.

2. Moorhead S, Johnson M, Mass ML, Swanson E. NOC: classificação dos resultados de enfermagem. Rio de Janeiro: Elsevier; 2010.

3. Hoskins LM. Clinical validation, methodologies for nursing diagnosis research. In: Carroll-Johnson RM. Classification of nursing diagnoses: proceedings of the eighth conference of North American Nursing Diagnosis Association. Philadelphia: JB Lippincott; 1989. p. 126-31.

4. Walker LO, Avant KC. Strategies for theory construction in nursing. 4th ed. Upper Saddle River: Pearson Prentice Hall; 2005.

5. Wilson J. Pensar com conceitos. 2. ed. São Paulo: Martins Fontes; 2005.

6. Lobiondo-Wood G, Haber J. Pesquisa em enfermagem: métodos, avaliação crítica e utilização. 4. ed. Rio de Janeiro: Guanabara Koogan; 2001.

7. Whittemore $R$, Knafl K. The integrative review: updated methodology. J Adv Nurs. 2005 Dec;52(5):546-53.

8. Melnyk BM, Fineout-Overholt E. Making the case for evidence-based practice. In: Melnyk BM, Fineout-Overholt E. Evidence-based practice in nursing \& healthcare: a guide to best practice. Philadelphia: Lippincot Williams\& Wilkins; 2005. p. 3-24.

9. Canning B, Sanchez G. Considering powered mobility for individuals with stroke. Top Stroke Rehabil. 2004
Spring; 11(2):84-8.

10. Stanko E, Goldie P, Nayler M. Development of a new mobility scale for people living in the community after stroke: content validity. Aust J Physiother. 2001;47(3):201-8.

11. Jutai J, Coulson S, Teasell R, Bayley M, Garland J, Mayo $\mathrm{N}$, et al. Mobility assistive device utilization in a prospective study of patients with first-ever stroke. Arch Phys Med Rehabil. 2007 Oct;88(10):1268-75.

12. Eng JJ, Rowe SJ, Mclaren LM. Mobility status during inpatient rehabilitation: a comparison of patients with stroke and traumatic brain injury. Arch Phys Med Rehabil. 2002 Apr;83(4):483-90.

13. van de Port IG, Kwakkel G, Schepers VP, Lindeman E. Predicting mobility outcome one year after stroke: a prospective cohort study. J Rehabil Med. 2006 Jul;38(4):218-23.

14. Mauritz KH. Gait training in hemiparetic stroke patients. Eura Medicophys. 2004 Sep;40(3):165-78.

15. Jette DU, Latham NK, Smout RJ, Gassaway J, Slavin MD, Horn SD. Physical therapy interventions for patients with stroke in inpatient rehabilitation facilities. Phys Ther. 2005 Mar;85(3):238-48.

16. Soares AV, Hochmuller ACOL, Silva P, Fronza D, Woellner SS, Noveletto F. Biorretroalimentação para treinamento do equilíbrio em hemiparéticos por acidente vascular encefálico: estudo preliminar. Fisioter Pesqui. 2009 Abr-Jun;16(2):132-6.

17. Engberg W, Lind A, Linder A, Nilsson L, Sernert N. Balance-related efficacy compared with balance function in patients with acute stroke. Physiother Theory Pract. 2008 Mar-Apr;24(2):105-11. 
18. Silveira CS, Zago MMF. Pesquisa brasileira em enfermagem oncológica: uma revisão integrativa. Rev Latinoam Enferm. 2006 Jul-Ago;14(4):614-9.

19. Mello ALLC, Coutinho ESF, Coeli CM. Prevalência de casos de acidente vascular encefálico, município do Rio de Janeiro. Cad Saúde Coletiva. 2006;14(2):345-60.
20. Tyson SF, Hanley M, Chillala J, Selley A, Tallis RC. Balance disability after stroke. Phys Ther. 2006 Jan;86(1):30-8.

21. Marin MJS, Rodrigues LCR, Druzian S, Cecílio LCO. Nursing diagnoses of elderly patients using multiple drugs. Rev Esc Enferm USP. 2010;44(1):46-51. 